\title{
Risk Assessment for Pulmonary Artery Hypertension in Adult Patient with Uncorrected Atrial Septal Defect by Six-Minute Walk Test and Mean Pulmonary Arterial Pressure
}

\author{
Mohammad Suryantoro*, Dyah Wulan Anggrahini, Anggoro Budi Hartopo, Lucia Kris Dinarti \\ Department of Cardiology and Vascular Medicine, Faculty of Medicine, Universitas Gadjah Mada - \\ Dr. Sardjito Hospital, Yogyakarta. Indonesia \\ *correspondence: surya.drrsu@gmail.com
}

\begin{abstract}
Background: Atrial septal defects (ASD) are the third most common type of congenital heart disease with an estimated incidence of 56 per 100,000 live births. Pulmonary Artery Hypertension (PAH) is one of thecomplication from ASD. Risk assessment in PAH can be determine by clinical patient, haemodynamic and exercise parameter. 6 Minute Walk Test (6MWT) is one of the exercise parameter risk assessment in $\mathrm{PAH}$. The aim of this study is to know correlation of 6MWT with mean Pulmonary Arterial Pressure (mPAP)in adult patient with uncorrected ASD.). 6MWT is one of the exercise test that measures and integrated response of all systems responsible during exercise. Pulmonary arterial hypertension $(\mathrm{PAH})$ is a problem encountered by patients with ASD. 6MWT is an objective tool to measure functional capacity of patients with PAH.

Method: A cross sectional study was performed to determine the walking distance in patients with ASD. We studied $265 \mathrm{PAH}$ in adult patient with uncorrected ASD at Sardjito Hospital, using the previous registry data (COHARD-PH (Congenital Heart Disease-Pulmonary Hypertension) registry). PAH were evaluated with right heart catheterization. 6MWT was collected. The correlation between 6MWT and MPAP was assessed by Spearman's correlation analysis using SPSS.

Result: We collected 265 patient PAH with uncorrected ASD (223 females (84\%) and 42 males $(16 \%)$, mean age $35.87 \pm 12.6$ years) underwent right heart catheterization. $31(11,7 \%), 217$ $(81,9 \%)$ and $17(6,4 \%)$ patients were in the low risk , intermediate and high risk for PAH according to 6MWT. 6MWT was correlated with mPAP ( $r=0.374$; $P<0.001)$.

Conclusion: The mPAP was correlated with 6MWT in patient with uncorrected ASD. A 6MWT can be useful to measure risk assessment of PAH in patient with ASD. Most of our study population represent intermediate risk for $\mathrm{PAH}$ according to 6MWT.
\end{abstract}

Keywords: atrial septal defect; pulmonary artery hypertension; 6MWT 